Divisão de Tuberculose. Centro de Vigilância Epidemiológica "Prof. Alexandre Vranjac". Coordenadoria de Controle de Doenças. Secretaria de Estado da Saúde. São Paulo, SP, Brasil

\section{Mudanças no tratamento da tuberculose}

\section{Changes in tuberculosis treatment}

Correspondência | Correspondence: Secretaria de Estado da Saúde de São Paulo Av. Dr. Arnaldo, $3511^{\circ}$ andar sala 135 01246-901 São Paulo, SP, Brasil E-mail: bepa@saude.sp.gov.br

Texto de difusão técnico-científica da Secretaria de Estado da Saúde de São Paulo.
Anualmente, cerca de 75.000 casos de tuberculose são notificados no Brasil. O Ministério da Saúde propôs um novo sistema de tratamento da doença, a partir de 2009. ${ }^{a}$ Basicamente, as mudanças ocorridas promovem: (1) a alteração das dosagens de pirazinamida e hidrazida; (2) a introdução de uma quarta droga, o etambutol, nos dois primeiros meses de tratamento; e (3) a formulação das quatro drogas num único comprimido, a dose fixa combinada. Assim, os esquemas vigentes até agora (I, II e de retratamento) serão substituídos pelo esquema básico, enquanto o esquema III deixará de ser utilizado.

De acordo com a Nota Técnica do Programa Nacional de Controle da Tuberculose (PNCT) do Ministério da Saúde, ${ }^{a}$ a mudança se justifica pela constatação do aumento da resistência primária à isoniazida (de 4,4\% para $6,0 \%$ ) e à isoniazida associada à rifampicina (de $1,1 \%$ para $1,4 \%$ ), observado no II Inquérito Nacional de Resistência aos Fármacos Anti-TB, realizado no período de 2007-2008, em comparação com os resultados do I Inquérito Nacional, conduzido em 1995 a 1997.

Essa alteração no esquema de tratamento contra a tuberculose ainda agrega benefícios como: redução do número de comprimidos a ser ingerido pelo doente, impossibilidade de tomada isolada das drogas e simplificação da gestão farmacêutica em todos os níveis.

Nesse primeiro momento, as mudanças ocorrerão apenas no tratamento dos doentes de tuberculose com mais de dez anos de idade. Para os indivíduos abaixo dessa faixa etária permanecem os esquemas vigentes atualmente no PNCT.

\section{TUBERCULOSE EM SÃO PAULO}

É importante conhecer em que contexto essas mudanças vão ocorrer em território paulista. Em 2008, foram notificados 19.143 casos de tuberculose em São Paulo - dos quais 15.759 novos -, com um coeficiente de incidência de 38,4 casos por 100.000 habitantes. Nos últimos 11 anos houve um declínio de $22,2 \%$ nas taxas de incidência, (Figura 1).

Quanto à mortalidade, em 2008 foram registrados no estado 724 óbitos por tuberculose (dados preliminares), com taxa de mortalidade de 1,77 óbitos por 100.000 habitantes, ou seja, um importante declínio de mais de $50 \%$ nos últimos 11 anos (Figura 2). Explicações para esse comportamento da doença em São Paulo podem ser,: a descentralização das ações de saúde no Estado, com a municipalização do atendimento; e,entre outras ações de controle, a implantação do tratamento supervisionado (DOT), a partir de 1996.

No entanto, a tuberculose impõe ainda grandes desafios à saúde pública paulista. Entre eles, o cumprimento da meta de $85 \%$ das taxas de cura no estado, ainda não atingida. Essa meta, por sua vez, está vinculada ao tratamento supervisionado, cuja cobertura está em torno de $50 \%$ dos casos.

Outro desafio importante refere-se aos fármacos utilizados no tratamento: ensaios clínicos em andamento, provavelmente, não identificarão novas drogas contra a tuberculose nos próximos dez anos. Daí a necessidade premente de "proteger" a rifampicina e a isoniazida da resistência.

\footnotetext{
a Ministério da Saúde. Secretaria de Vigilância em Saúde. Departamento de Vigilância Epidemiológica. Programa Nacional de Controle da Tuberculose. Nota técnica sobre as mudanças no tratamento da tuberculose no Brasil para adultos e adolescentes. Brasília; 2009 [citado 2009 dez 10]. Disponível em: http://portal.saude.gov.br/portal/arquivos/pdf/nota_tecnica_versao_28_de_agosto_v_5.pdf
} 
Tabela 1. Esquema básico para adultos e adolescentes.

\begin{tabular}{lcccc}
\hline Regime & Fármaco & Faixa de peso & Unidades/dose & Duração (meses) \\
\hline \multirow{2}{*}{$\begin{array}{l}\text { RHZE } \\
\text { Fase intensiva }\end{array}$} & $\begin{array}{l}\text { RHZE } \\
\text { comprimido em dose } \\
\text { fixa combinada }\end{array}$ & $>50 \mathrm{~kg}$ & 2 comprimidos & 2 \\
4RH & $\mathrm{RH}$ & 20 a $35 \mathrm{~kg}$ & 4 comprimidos & \\
Fase de manutenção & $300 / 200$ ou 150/100 & 36 a $50 \mathrm{~kg}$ & 1 cáps $300 / 200+1$ cáp $150 / 100$ & 4 \\
& cápsula & $>50 \mathrm{~kg}$ & 2 cápsulas 300/200 & \\
\hline
\end{tabular}

Fonte: Programa Nacional de Controle da Tuberculose do Ministério da Saúde

R: Rifampicina; H: Isoniazida; Z: Pirazinamida; E: Etambutol

\section{ESQUEMA BÁSICO}

A mudança proposta pelo Ministério da Saúde deverá ocorrer paulatinamente, de acordo com as estratégias de cada Estado. No caso de São Paulo, a implantação teve início em setembro de 2009, com treinamento dos profissionais de saúde envolvidos nas ações de controle da tuberculose e culminando com a disponibilização das drogas nos serviços de saúde, entre 7 e 15 de dezembro.

O esquema básico será composto, nos dois primeiros meses, pelo Coxcip 4 (comprimido contendo em dose fixa combinada rifampicina, isoniazida, pirazinamida e etambutol); e nos quatro últimos meses pela rifampicina e isoniazida (cápsula contendo $300 \mathrm{mg}$ de rifampicina e $200 \mathrm{mg}$ de isoniazida), esquema já utilizado na rede pública de saúde.

Um adulto com peso de 50 quilos deverá tomar quatro comprimidos de Coxcip 4 nos dois primeiros meses, e na fase de manutenção (quatro meses seguintes), duas cápsulas de rifampicina e isoniazida

\section{INDICAÇÕES}

O esquema básico é indicado aos casos novos (paciente que nunca usou ou usou por menos de 30 dias medicamentos antituberculose) de todas as formas de tuberculose pulmonar e extrapulmonar (exceto meningoencefalite), infectados ou não pelo vírus HIV; e no retratamento. Em todos os casos de retratamento, preconiza-se a solicitação de cultura, identificação e teste de sensibilidade.

O esquema básico para o tratamento da tuberculose e da meningoencefalite está exposto nas Tabelas 1 e 2 .

Recomenda-se a solicitação de cultura, identificação e teste de sensibilidade (TS) para todos os casos com baciloscopia positiva ao final do segundo mês de tratamento. Com o resultado do TS, que permite identificar a resistência às drogas, a unidade de referência pode avaliar uma eventual mudança do esquema de tratamento. Até o retorno e avaliação do TS deverá ser mantido o esquema inicial.

Na meningoencefalite tuberculosa deve ser associado corticosteróide ao esquema anti-TB: prednisona oral (1-2 $\mathrm{mg} / \mathrm{kg} / \mathrm{dia})$ por quatro semanas ou dexametasona intravenoso nos casos graves ( $0.3 \mathrm{a} 0.4 \mathrm{mg} / \mathrm{kg} / \mathrm{dia})$, por 4-8 semanas, com redução gradual da dose nas quatro semanas subseqüentes.

Os profissionais de saúde, responsáveis pela organização dos serviços, devem lembrar que haverá a necessidade de conviver, por um período, com os esquemas anteriormente adotados e o esquema básico.

As mudanças incluem também a estrutura da rede assistencial, com proposta de consolidar uma rede hierarquizada para atendimento aos pacientes portadores da tuberculose. A porta de entrada para os casos novos e retratamentos será, sempre que possível, a rede básica do Sistema Único de Saúde. Os casos que

Tabela 2. Esquema para meningoencefalite para adultos e adolescentes.

\begin{tabular}{lcccc}
\hline Regime & Fármaco & Faixa de peso & Unidades/dose & Duração (meses) \\
\hline RHHZE & RHZE & 20 a $35 \mathrm{~kg}$ & 2 comprimidos & \\
Fase intensiva & $150 / 75 / 400 / 275$ & 36 a $50 \mathrm{~kg}$ & 3 comprimidos & 2 \\
& $\begin{array}{c}\text { comprimido em dose } \\
\text { fixa combinada }\end{array}$ & $>50 \mathrm{~kg}$ & 4 comprimidos & 7 \\
7 RH & RH & 20 a $35 \mathrm{~kg}$ & 1 cápsula $300 / 200$ & \\
Fase de manutenção & $300 / 200$ ou 150/100 & 36 a $50 \mathrm{~kg}$ & 1 cáps 300/200 + 1 cáp 150/100 & \\
& cápsula & $>50 \mathrm{~kg}$ & 2 cápsulas 300/200 & \\
\hline
\end{tabular}

Fonte: Programa Nacional de Controle da Tuberculose do Ministério da Saúde 


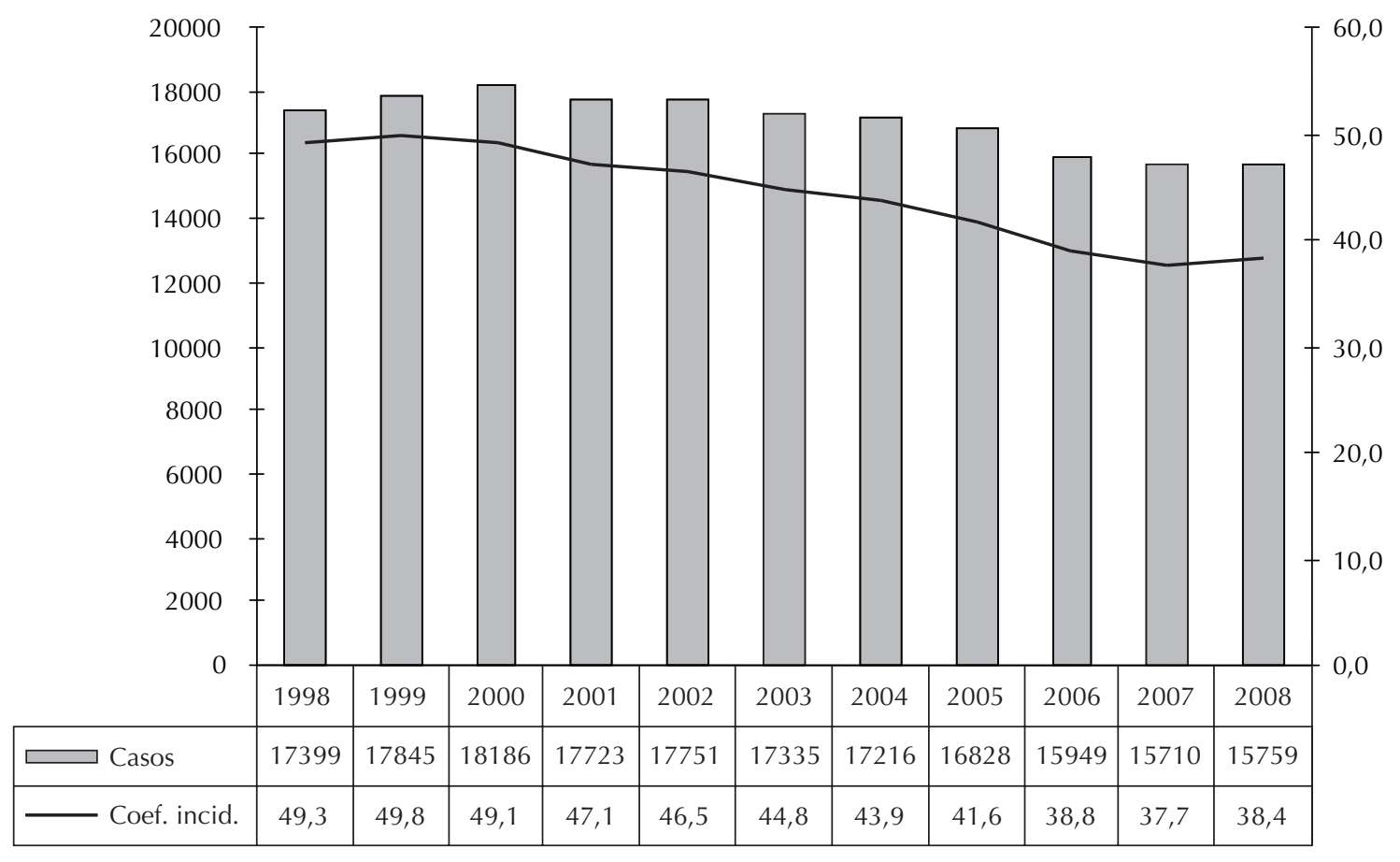

Figura 1. Casos e coeficientes de incidência de tuberculose. Estado de São Paulo, 1998 a 2008.

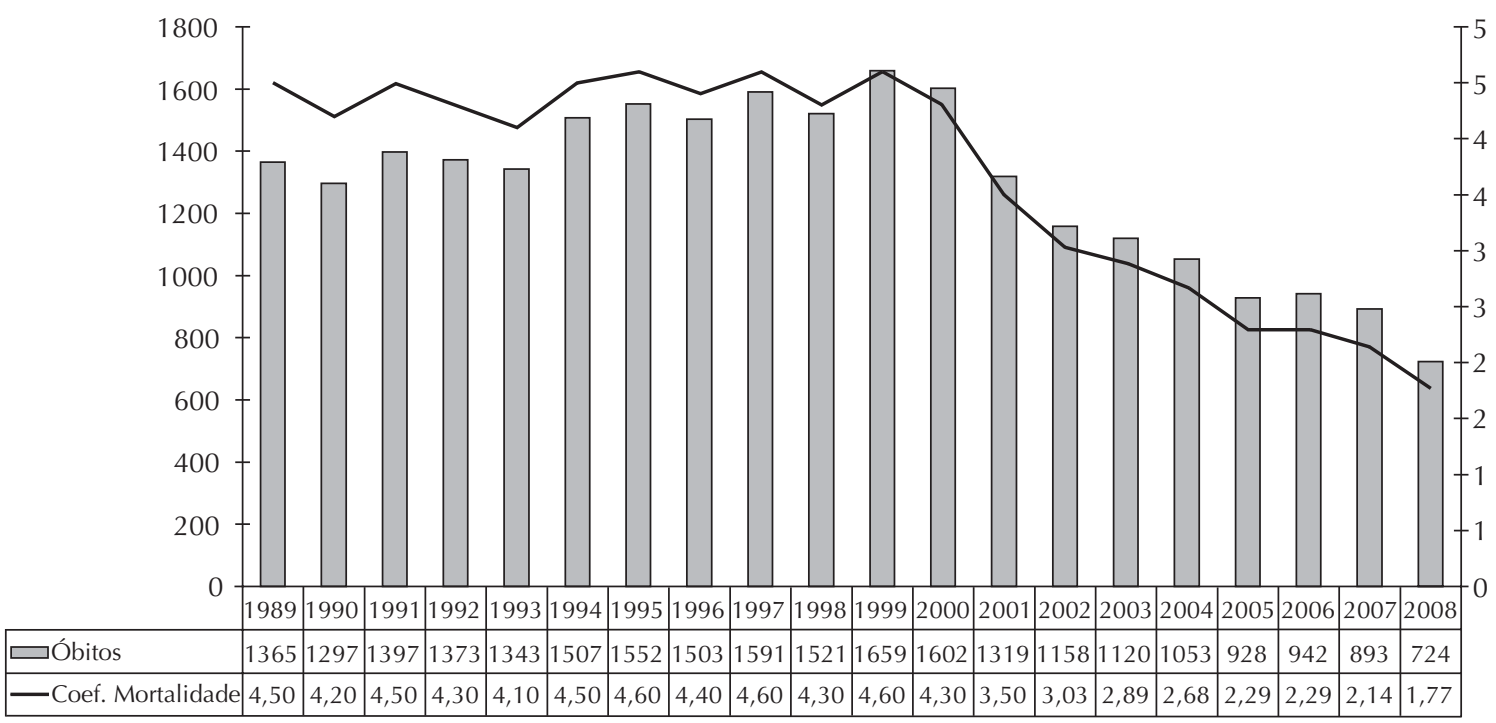

Figura 2. Óbitos e coeficientes de mortalidade por tuberculose. Estado de São Paulo, 1989 a 2008.

necessitarem de elucidação diagnóstica e apresentarem efeitos adversos maiores aos tuberculostáticos deverão ser encaminhados aos ambulatórios de referência secundária e aqueles com alguma resistência às drogas, aos ambulatórios de referência para casos resistentes.

O estado de São Paulo conta, hoje, com cerca de 60 ambulatórios de referência secundária, além de sete ambulatórios de referência para tratamento de pacientes com resistência às drogas e três hospitais de longa permanência que também internam casos de tuberculose com multirresistência. A lista desses serviços, com endereço e telefone, está disponível no site da Divisão de Tuberculose do Centro de Vigilância Epidemiológica "Prof. Alexandre Vranjac" (http://www.cve.saude. sp.gov.br). 